\title{
Dynamical Systems
}

\section{and \\ Quantum Bicrossproduct Algebras}

\author{
Oscar Arratia ${ }^{1}$ and Mariano A. del $\mathrm{Olmo}^{2}$ \\ 1 Departamento de Matemática Aplicada a la Ingeniería, \\ 2 Departamento de Física Teórica, \\ Universidad de Valladolid, E-47011, Valladolid, Spain \\ E. mail: oscarr@wmatem.eis.uva.es, olmo@fta.uva.es
}

November 1, 2018

\begin{abstract}
We present a unified study of some aspects of quantum bicrossproduct algebras of inhomogeneous Lie algebras, like Poincaré, Galilei and Euclidean in $N$ dimensions. The action associated to the bicrossproduct structure allows to obtain a nonlinear action over a new group linked to the translations. This new nonlinear action associates a dynamical system to each generator which is the object of study in this paper.
\end{abstract}




\section{Introduction}

In a series of papers [1]-[5] we have dealt with the problem of the construction of induced representations of quantum inhomogeneous algebras. In particular, in Ref. [1 we have focussed our attention on quantum Hopf algebras having the structure of bicrossproduct $\mathcal{H}=U(\mathcal{K}) \bowtie U_{z}(\mathcal{L})$, with $U(\mathcal{K})$ a cocommutative Hopf algebra, $U_{z}(\mathcal{L})$ a commutative but noncocommutative Hopf algebra and $\mathcal{K}$ and $\mathcal{L}$ Lie algebras [6]. Remember that this bicrossproduct structure is the deformed counterpart of the semidirect product of Lie groups $(H=L \odot K)$. In this paper we want to profit of some of the techniques developed in the above mentioned papers to obtain relevant information about some aspects related with the bicrossproduct algebras object of our study.

We shall reinterpret the above bicrossproduct structure as $H=U(\mathcal{K}) \triangleright \triangleleft \operatorname{Fun}\left(L_{z}\right)$, because the commutativity of $\mathcal{L}$ allows to identify it with the algebra of functions over a certain group $L_{z}$. The bicrossproduct structure determines an action of $U(\mathcal{K})$ on $\operatorname{Fun}\left(L_{z}\right)$ which at the level of groups originates a nonlinear action of the group $K$ on $L_{z}$. At the infinitesimal level this last action is described by vector fields associated to the generators of $\mathcal{K}$. These vector fields give rise to oneparameter flows some of them linear ('nondeformed') and other nonlinear ('deformed'). In other words, we can study some dynamical systems associated to this action.

As it is well known in the nondeformed case, the homogeneous space $X=H / K$ is diffeomorphic to $\mathbb{R}^{N}$ which is associated to $L$, being $N$ the dimension of $L$. In the quantum case the homogeneous space is now identified with $\operatorname{Fun}\left(L_{z}\right)$. However, we can study the nonlinear action of $K$ over $L_{z}$. Note that in the limit $z \rightarrow 0$ we recover the linear action of $K$ on $X$.

We will consider the family of inhomogeneous algebras related by graded contractions with the compact algebra $s o(N+1)$ [7, 8]. They are called inhomogeneous Cayley-Klein algebras. Among the elements of this family we find the Poincaré and the Galilei algebras in $(N-1,1)$ dimensions and the Euclidean algebra in $N$ dimensions. The bicrossproduct structure that share these quantum algebras [9] allows to present a unified study of the properties mentioned above.

The organization of the paper is as follows. Section 2 presents a brief review about the inhomogeneous Cayley-Klein algebras, their quantum deformations and their bicrossproduct structure. Next section, the most important of the work, is devoted to compute the flow associated to the action, the invariant under this action that coincides with the Casimir, and the dynamical systems associated. In section 10 we present, as an example, the case of $N=3$ to illustrate the ideas introduced in the previous section. We finish with some conclusions and remarks.

\section{Quantum Cayley-Klein algebras $U_{z}\left(\mathfrak{i s o}_{\omega_{2}, \omega_{3}, \ldots, \omega_{N}}(N)\right)$}

The family of Cayley-Klein pseudo-orthogonal algebras is a set of $(N+1) N / 2$ dimensional real Lie algebras characterized by $N$ real parameters $\left(\omega_{1}, \omega_{2}, \ldots, \omega_{N}\right)$ and denoted $\mathfrak{s o}_{\omega_{1}, \omega_{2}, \ldots, \omega_{N}}(N+1)$ [4], 8]. In an appropriate basis $\left(J_{i j}\right)_{0 \leq i<j \leq N}$ the nonvanishing commutators are

$$
\left[J_{i j}, J_{i k}\right]=\omega_{i j} J_{j k}, \quad\left[J_{i j}, J_{j k}\right]=-J_{i k}, \quad\left[J_{i k}, J_{j k}\right]=\omega_{j k} J_{i j},
$$

with the subindices verifying $0<i<j<k<N$ and $\omega_{i j}=\prod_{s=i+1}^{j} \omega_{s}$. The generators can be rescaled in such a way that the parameters $\omega_{i}$ only take the values 1,0 and -1 . When all the $\omega_{i}$ 's 
are different to zero the algebra $\mathfrak{s o}_{\omega_{1}, \omega_{2}, \ldots, \omega_{N}}(N+1)$ is isomorphic to some of the pseudo-orthogonal algebras $\mathfrak{s o}(p, q)$ with $p+q=N+1$ and $p \geq q>0$. If some of the coefficients $\omega_{i}$ vanishes the corresponding algebra is inhomogeneous and can be obtained from $\mathfrak{s o}(p, q)$ by means of a sequence of contractions. In the particular case of $\omega_{1}=0$, the algebras $\mathfrak{s o}_{0, \omega_{2}, \ldots, \omega_{N}}(N+1)$ can be realized as algebras of groups of affine transformations on $\mathbb{R}^{N}\left[\right.$ [7]. In this case, the generators $J_{0 i}$ are denoted by $P_{i}$ stressing, in this way, its role as generators of translations. The remaining generators $J_{i j}$ originate compact and 'noncompact' rotations. These inhomogeneous algebras, henceforth denoted by $\mathfrak{i s o}_{\omega_{2}, \ldots, \omega_{N}}(N)$, are characterized by the following nonvanishing commutators

$$
\begin{array}{llr}
{\left[J_{i j}, J_{i k}\right]=\omega_{i j} J_{j k},} & {\left[J_{i j}, J_{j k}\right]=-J_{i k},} & {\left[J_{i k}, J_{j k}\right]=\omega_{j k} J_{i j},} \\
{\left[J_{i j}, P_{i}\right]=P_{j},} & {\left[J_{i j}, P_{i}\right]=-\omega_{i j} P_{i},} & 1 \leq i<j<k \leq N .
\end{array}
$$

In [10, 11] simultaneous standard deformations (i.e. their associated classical $r$-matrices are quasi-triangular [12]) for all the enveloping algebras $U\left(\mathfrak{s o}_{\omega_{1}, \omega_{2}}(3)\right)$ and $U\left(\mathfrak{s o}_{\omega_{1}, \omega_{2}, \omega_{3}}(4)\right)$, respectively, were introduced. In 13] the case of $U\left(\mathbf{i s o}_{\omega_{2}, \omega_{3}, \omega_{4}}(4)\right)$ was considered, and in 14 the general case $U\left(\mathfrak{i s o}_{\omega_{2}, \omega_{3}, \ldots, \omega_{N}}(N)\right)$ (all of them standard deformations).

It was proved in [9] that the standard quantum Hopf algebras $U_{z}\left(\mathfrak{i s o}_{\omega_{2}, \omega_{3}, \ldots, \omega_{N}}(N)\right)$ have a structure of bicrossproduct. Using a basis adapted to the bicrossproduct structure we can describe together all these quantum algebras $U_{z}\left(\mathfrak{i s c}_{\omega_{2}, \omega_{3}, \ldots, \omega_{N}}(N)\right)$. In order to avoid repetitions we use the following convention: the variation rank of $i, j, k$ is $1, \ldots, N-1$ and the index $N$ is treated separately. Besides, when two indices $i, j$ appear in a generator it is assumed that $i<j$. The commutation relations are

$$
\begin{array}{lll}
{\left[P_{i}, P_{j}\right]=0,} & {\left[P_{i}, P_{N}\right]=0,} & \\
{\left[J_{i j}, J_{i k}\right]=\omega_{i j} J_{j k},} & {\left[J_{i j}, J_{j k}\right]=-J_{i k},} & {\left[J_{i k}, J_{j k}\right]=\omega_{j k} J_{i j},} \\
{\left[J_{i j}, J_{i N}\right]=\omega_{i j} J_{j N},} & {\left[J_{i j}, J_{j N}\right]=-J_{i N},} & {\left[J_{i k}, J_{j N}\right]=\omega_{j N} J_{i j},} \\
{\left[J_{i j}, P_{k}\right]=\delta_{i k} P_{k}-\delta_{j k} \omega_{i j} P_{i},} & {\left[J_{i j}, P_{N}\right]=0,} \\
{\left[J_{i N}, P_{j}\right]=\delta_{i j}\left(\frac{1-e^{-2 z P_{N}}}{2 z}-\frac{z}{2} \sum_{s=1}^{N-1} \omega_{s N} P_{s}^{2}\right)+z \omega_{i N} P_{i} P_{j},} & {\left[J_{i N}, P_{N}\right]=-\omega_{i N} P_{i} ;}
\end{array}
$$

and the coproduct is given by

$$
\begin{aligned}
& \Delta\left(P_{i}\right)=P_{i} \otimes 1+e^{-z P_{N}} \otimes P_{i}, \quad \Delta\left(P_{N}\right)=P_{N} \otimes 1+1 \otimes P_{N}, \\
& \Delta\left(J_{i j}\right)=J_{i j} \otimes 1+1 \otimes J_{i j}, \\
& \Delta\left(J_{i N}\right)=J_{i N} \otimes 1+e^{-z P_{N}} \otimes J_{i j}+z \sum_{s=1}^{i-1} \omega_{i N} P_{s} \otimes J_{s i}-z \sum_{s=i+1}^{N-1} \omega_{s N} P_{s} \otimes J_{i s} .
\end{aligned}
$$

The bicrossproduct structure $U_{z}\left(\mathfrak{i s o}_{\omega_{2}, \omega_{3}, \ldots, \omega_{N}}(N)\right)=\mathcal{K} \bowtie \mathcal{L}$, with $\mathcal{K}=U\left(\mathfrak{s o}_{\omega_{2}, \omega_{3}, \ldots, \omega_{N}}(N)\right)$ and $\mathcal{L}$ the commutative Hopf subalgebra generated by $P_{1}, P_{2}, \ldots, P_{N}$, is described by the right action of $\mathcal{K}$ over $\mathcal{L}$

$$
P_{i} \triangleleft J_{j k}=\left[P_{i}, J_{j k}\right], \quad j<k, i, j, k=1,2, \ldots, N,
$$


with the commutators given by (2.1), and the left coaction of $\mathcal{L}$ over $\mathcal{K}$, whose expression over the generators of $\mathcal{K}$ is

$$
\begin{aligned}
& J_{i j} \triangleleft=1 \otimes J_{i j}, \\
& J_{i N} \triangleleft=e^{-z P_{N}} \otimes J_{i N}+z \sum_{s=1}^{i-1} \omega_{i N} P_{s} \otimes J_{s i}-z \sum_{s=i+1}^{N-1} \omega_{s N} P_{s} \otimes J_{i s} .
\end{aligned}
$$

\section{Oneparameter flows}

In [9] the algebra $U_{z}\left(T_{N}\right)$ was considered as a noncommutative deformation of the Lie algebra of the group of translations of $\mathbb{R}^{N}$. However, here we can profit the commutativity of $U_{z}\left(T_{N}\right)$ for interpreting it as the algebra of functions over a group, in such a way that we have the following bicrossproduct decomposition

$$
U_{z}\left(\mathfrak{i s o}_{\omega_{2}, \omega_{3}, \ldots, \omega_{N}}(N)\right)=U\left(\mathfrak{s o}_{\omega_{2}, \omega_{3}, \ldots, \omega_{N}}(N)\right) \bowtie F\left(T_{z, N}\right),
$$

where $T_{z, N}$ is the space $\mathbb{R}^{N}$ equipped with the composition law

$$
\begin{aligned}
& \left(\alpha_{1}^{\prime}, \alpha_{2}^{\prime}, \ldots, \alpha_{N-1}^{\prime}, \alpha_{N}^{\prime}\right)\left(\alpha_{1}, \alpha_{2}, \ldots, \alpha_{N-1}, \alpha_{N}\right)= \\
& \left(\alpha_{1}^{\prime}+e^{-z \alpha_{N}^{\prime}} \alpha_{1}, \alpha_{2}^{\prime}+e^{-z \alpha_{N}^{\prime}} \alpha_{2}, \ldots, \alpha_{N-1}^{\prime}+e^{-z \alpha_{N}^{\prime}} \alpha_{N-1}, \alpha_{N}^{\prime}+\alpha_{N}\right),
\end{aligned}
$$

that equips it with a structure of $N$-dimensional Lie group. The group $T_{z, N}$ has the structure of semidirect product of the additive groups $\mathbb{R}^{N-1}$ and $\mathbb{R}$

$$
T_{z, N} \equiv \mathbb{R}^{N-1} \rtimes \mathbb{R}, \quad\left(a^{\prime}, b^{\prime}\right)(a, b)=\left(a^{\prime}+a \triangleleft b^{\prime-1}, b^{\prime}+b\right),
$$

where the right action of $\mathbb{R}$ over $\mathbb{R}^{N-1}$ is given by means of the usual product over each component,

$$
a \triangleleft b=e^{z b} a, \quad a \in \mathbb{R}^{N-1}, \quad b \in \mathbb{R} .
$$

The generators $P_{i}$ of $U_{z}\left(T_{N}\right)$ give in this context a global chart over $T_{z, N}$,

$$
P_{i}(\alpha)=\alpha_{i}, \quad \alpha \in T_{z, N}
$$

The structure of $U\left(\mathfrak{s o}_{\omega_{2}, \omega_{3}, \ldots, \omega_{N}}(N)\right)$-module algebra of $F\left(T_{z, N}\right)$ implies that an action of the group $S O_{\omega_{2}, \omega_{3}, \ldots, \omega_{N}}(N)$ on $T_{z, N}$ is defined. At the infinitesimal level this action is described by the vector fields

$$
\begin{aligned}
\hat{J}_{i j} & =-P_{j} \frac{\partial}{\partial P_{i}}+\omega_{i j} P_{i} \frac{\partial}{\partial P_{j}}, \\
\hat{J}_{i N} & =\sum_{j=1}^{N-1}-\left[\delta_{i j}\left(\frac{1-e^{-2 z P_{N}}}{2 z}-\frac{z}{2} \sum_{s=1}^{N-1} \omega_{s N} P_{s}^{2}\right)+z \omega_{i N} P_{i} P_{j}\right] \frac{\partial}{\partial P_{j}}+\omega_{i N} P_{i} \frac{\partial}{\partial P_{N}} \\
& =\sum_{j \neq i, N}-z \omega_{i N} P_{i} P_{j} \frac{\partial}{\partial P_{j}}-\left[\frac{1-e^{-2 z P_{N}}}{2 z}-\frac{z}{2} \sum_{s=1}^{N-1} \omega_{s N} P_{s}^{2}+z \omega_{i N} P_{i}^{2}\right] \frac{\partial}{\partial P_{i}}+\omega_{i N} P_{i} \frac{\partial}{\partial P_{N}} .
\end{aligned}
$$


Since only the generators $J_{1 N}, J_{2 N}, \ldots, J_{N-1}$ have deformed action the integration of the equations of the fields $\hat{J}_{i j}$ is immediate and gives the well known linear flows

$$
\Phi_{i j}^{t}\left(\alpha_{1}, \ldots, \alpha_{i}, \ldots, \alpha_{j}, \ldots, \alpha_{N}\right)=\left(\alpha_{1}, \ldots, \alpha_{i-1}, \alpha_{i}^{\prime}, \alpha_{i+1} \ldots, \alpha_{j-1}, \alpha_{j}^{\prime}, \alpha_{j+1} \ldots, \alpha_{N}\right),
$$

with

$$
\alpha_{i}^{\prime}=C_{\omega_{i j}}(t) \alpha_{i}-S_{\omega_{i j}}(t) \alpha_{j}, \quad \alpha_{j}^{\prime}=\omega_{i j} S_{\omega_{i j}}(t) \alpha_{i}+C_{\omega_{i j}}(t) \alpha_{j}
$$

where

$$
C_{\omega}(t)=\frac{e^{\sqrt{-\omega} t}+e^{-\sqrt{-\omega} t}}{2}, \quad S_{\omega}(t)=\frac{e^{\sqrt{-\omega} t}-e^{-\sqrt{-\omega} t}}{2 \sqrt{-\omega}}
$$

So, we have simple compact or noncompact rotations in the $i j$ plane.

The computation of the flows associated to the 'deformed' fields $\hat{J}_{i N}$ requires a more careful analysis. Let us start by obtaining their invariants. Supposing that the differential form

$$
\eta=\sum_{s=1}^{N} \mu_{s} d P_{s}
$$

verifies $\left.\hat{J}_{i N}\right\rfloor \eta=0$, the following equation is obtained

$$
\sum_{j \neq i, N} z \omega_{i N} P_{i} P_{j} \mu_{j}+\left[\frac{1-e^{-2 z P_{N}}}{2 z}-\frac{z}{2} \sum_{s=1}^{N-1} \omega_{s N} P_{s}^{2}+z \omega_{i N} P_{i}^{2}\right] \mu_{i}-\omega_{i N} P_{i} \mu_{N}=0 .
$$

Using this expression $(N-1)$ invariant functions are obtained as follows. For the first invariant we choose

$$
\mu_{s}=\omega_{s N} P_{s} \tau, \quad s=1,2, \ldots, N-1,
$$

with $\tau$ a function to be evaluated. Hence, equation (3.4) reduces to

$$
\omega_{i N} P_{i}\left[\frac{1-e^{-2 z P_{N}}}{2 z}+\frac{z}{2} \sum_{s=1}^{N-1} \omega_{s N} P_{s}^{2}\right] \tau-\omega_{i N} P_{i} \mu_{N}=0 .
$$

From equation (3.5) we find the value of $\mu_{N}$ obtaining the differential form

$$
\eta=\tau\left[\sum_{j=1}^{N-1} \omega_{j N} P_{j} d P_{j}+\left(\frac{1-e^{-2 z P_{N}}}{2 z}+\frac{z}{2} \sum_{s=1}^{N-1} \omega_{s N} P_{s}^{2}\right) d P_{N}\right],
$$

where $\tau$ plays the role of integration factor. Solving the case $N=2$ we get $\tau=2 e^{z P_{N}}$, that it is proved to be valid for every $N$. The integration of the equations

$$
\frac{\partial h}{\partial P_{s}}=\mu_{s}, \quad 1 \leq s \leq N,
$$

gives $\eta=d h$. By an appropriate choice of the integration constant, in order to have a well behaviour in the limit $z \rightarrow 0$, we obtain

$$
h_{\omega, z}=\sum_{j=1}^{N-1} \omega_{j N} P_{j}^{2} e^{z P_{N}}+\frac{\cosh \left(z P_{N}\right)-1}{\frac{z^{2}}{2}} .
$$


This function is, in fact, invariant under the action of all the generators $J_{i j}$. Indeed, it belongs to the center of the algebra $U_{z}\left(\mathfrak{i s o}_{\omega_{2}, \omega_{3}, \ldots, \omega_{N}}(N)\right)$ and is the Casimir $C_{z}$ given in [9], but now it appears in a natural way.

To obtain the other $N-2$ invariants we start fixing $k \in\{1,2, \ldots, N-1\}-\{i\}$ and taking $\mu_{j}=0$ if $j \neq k, N$, we get from expression (3.3) the differential form $\eta_{k}=\mu_{k} d P_{k}+\mu_{N} d P_{N}$. Condition (3.4) applied to $\eta_{k}$ establishes a relationship between $\mu_{k}$ and $\mu_{N}$ that allows to write

$$
\eta_{k}=\mu_{k} d P_{k}+\mu_{k} z P_{k} d P_{N}
$$

Choosing $\mu_{k}=e^{z P_{N}}$ the differential form is exact, that is, $\eta_{k}=d h_{\omega, z}^{i N, k}$, with

$$
h_{\omega, z}^{i N, k}=P_{k} e^{z P_{N}}, \quad k \in\{1,2, \ldots, N-1\}-\{i\} .
$$

To obtain the integral curves of $\hat{J}_{i N}$ it is necessary to solve the system of $N$ differential equations

$$
\begin{aligned}
& \dot{\alpha}_{j}=-z \omega_{i N} \alpha_{i} \alpha_{j}, \quad j \neq i, N, \\
& \dot{\alpha}_{i}=-\frac{1-e^{-2 z \alpha_{n}}}{2 z}+\frac{z}{2} \sum_{s=1}^{N-1} \omega_{s N} \alpha_{s}^{2}-z \omega_{i N} \alpha_{i}^{2}, \\
& \dot{\alpha}_{N}=\omega_{i N} \alpha_{i} .
\end{aligned}
$$

The invariants $h_{\omega, z}^{i N, k}(3.7)$ allow to remove $N-2$ degrees of freedom, from $h_{\omega, z}^{i N, k}(\alpha)=\alpha_{k} e^{z \alpha_{N}}=\beta_{k}$ we obtain $\alpha_{k}=\beta_{k} e^{-z \alpha_{N}}$, restricting the study of the $N$-dimensional system to the following family of 2-dimensional systems depending on the $N$ parameters $\beta_{k}, \omega$ and $z$

$$
\begin{aligned}
& \dot{\alpha}_{i}=-\left[\frac{1-e^{-2 z \alpha_{N}}}{2 z}-\frac{z}{2}\left(\sum_{s \neq i, N} \omega_{s N} \beta_{s}^{2}\right) e^{-2 z \alpha_{N}}+\frac{z}{2} \omega_{i N} \alpha_{i}^{2}\right] \\
& \dot{\alpha}_{N}=\omega_{i N} \alpha_{i} .
\end{aligned}
$$

Due to the way in which the parameters $\beta_{k}$ appear grouped, the set of systems (3.8) only depends on three parameters $z, \omega_{i N}$ and $\rho=\sum_{s \neq i, N} \omega_{s N} \beta_{s}^{2}$. The function $h_{\omega, z}$ (3.6) gives the following invariant for the system (3.8)

$$
\omega_{i N} \alpha_{i}^{2} e^{z \alpha_{N}}+\rho e^{-z \alpha_{N}}+\frac{\cosh \left(z \alpha_{N}\right)-1}{\frac{z^{2}}{2}} .
$$

The description of the systems when $z=0$ is trivial, since it reduces to the study of linear systems analogue to those of the fields $\hat{J}_{i j}$. If $z$ does not vanish the equations may be rescaled considering

$$
x(t)=z \alpha_{i}(t), \quad y(t)=z \alpha_{N}(t),
$$

and setting $a=\omega_{i N}, b-1=z^{2} \rho=z^{2} \sum_{s \neq i, N} \omega_{s N} \beta_{s}^{2}$ the system becomes

$$
\dot{x}=-\frac{1}{2} a x^{2}-\frac{1}{2}+\frac{1}{2} b e^{-2 y}, \quad \dot{y}=a x .
$$


In this form the limit $z \rightarrow 0$ cannot be studied, but in advantage it depends on only two parameters. The possibility of reabsortion of the parameter $z$ is followed from the fact that all the Hopf algebras $U_{z}\left(\mathfrak{i s o}_{\omega_{2}, \omega_{3}, \ldots, \omega_{N}}(N)\right)$ are isomorphic (for fixed values of the parameters $\omega_{s}$ ) whenever $z$ is nonzero. The function (3.9) gives rise to the following invariant of (3.10)

$$
h_{a, b}=a x^{2} e^{y}+e^{y}+b e^{-y} .
$$

The research of fixed points of the system reveals that:

- if $b \leq 0$ the system has not equilibrium points;

- if $b>0$ there are three possibilities:

- if $a<0$ then there is only one fixed point $\left(0, \frac{1}{2} \ln b\right)$ of hyperbolic character,

- if $a=0$ then all the points like $\left(x, \frac{1}{2} \ln b\right)$ are fixed points,

- if $a>0$ there is only one equilibrium point $\left(0, \frac{1}{2} \ln b\right)$ of elliptic character.

Let us go to analyze in detail the case $a>0$ and $b>0$. Here the invariant (3.11) has a global minimum of value $2 \sqrt{b}$ at $\left(0, \frac{1}{2} \ln b\right)$ and it is easy to check that $h_{a, b}$ takes arbitrarily high values over points going to infinity in any direction. Since the orbits of the system (3.10) are the level curves of $h_{a, b}$ all the orbits are bounded. Note that the point of equilibrium disappears in the limit $b \rightarrow 0$. Let us consider the integral curve $\gamma_{r}$ passing through the point $(0, r)$, with $r>\frac{1}{2} \ln b$, at the initial time. For small values of $t>0$ the invariant allows to obtain $x$ in terms of $y$

$$
a x=-\sqrt{a e^{-y}\left(e^{r}+b e^{-r}-e^{y}-b e^{-y}\right)},
$$

in such a way that substituting in the second of the equations of the system 3.10) it is enough to do a quadrature. The final result gives the following expression for the curve $\gamma_{r}$

$$
\gamma_{r}(t)=\left(\frac{-\left(e^{r}-b e^{-r}\right) S_{a}(t)}{\left(e^{r}+b e^{-r}\right)+\left(e^{r}-b e^{-r}\right) C_{a}(t)}, \ln \frac{1}{2}\left[\left(e^{r}+b e^{-r}\right)+\left(e^{r}-b e^{-r}\right) C_{a}(t)\right]\right) .
$$

From (3.13) the flow associated to the system (3.10) is obtained supposed $a>0$ and $b>0$

$$
\begin{aligned}
\Phi_{a, b}^{t}(x, y)= & \left(\frac{\left(a x^{2} e^{y}-e^{y}+b e^{-y}\right) S_{a}(t)+\left(2 x e^{y}\right) C_{a}(t)}{\left(a x^{2} e^{y}+e^{y}+b e^{-y}\right)+\left(-a x^{2} e^{y}+e^{y}-b e^{-y}\right) C_{a}(t)+\left(2 a x e^{y}\right) S_{a}(t)},\right. \\
& \left.\ln \frac{1}{2}\left[\left(a x^{2} e^{y}+e^{y}+b e^{-y}\right)+\left(-a x^{2} e^{y}+e^{y}-b e^{-y}\right) C_{a}(t)+\left(2 a x e^{y}\right) S_{a}(t)\right]\right) .
\end{aligned}
$$

It is immediate to prove that (3.14) is also correct for the remaining values of $a$ and $b$. However, if the parameters $a$ and $b$ are positive the flow is defined globally, but this does not happen, in general, for any other value of the parameters.

The preceding study allows to write the flow $\Phi_{i N}^{t}: T_{z, N} \rightarrow T_{z, N}$ of the vector field $\hat{J}_{i N}$ (3.1). For its description it is convenient to introduce the functions $F_{i N}^{\omega, z}: T_{z, N} \times \mathbb{R} \rightarrow \mathbb{R}$, defined by

$$
\begin{aligned}
F_{i N}^{\omega, z}(\alpha, t)= & {\left[\cosh \left(z \alpha_{N}\right)+\frac{z^{2}}{2} \sum_{s=1}^{N-1} \omega_{s N} \alpha_{s}^{2} e^{z \alpha_{N}}\right] } \\
& +\left[\sinh \left(z \alpha_{N}\right)-\frac{z^{2}}{2} \sum_{s=1}^{N-1} \omega_{s N} \alpha_{s}^{2} e^{z \alpha_{N}}\right] C_{\omega_{i N}}(t)+\left[z \omega_{i N} \alpha_{i} e^{z \alpha_{N}}\right] S_{\omega_{i N}}(t) .
\end{aligned}
$$


Note that the first term can be written in terms of the invariant $h_{\omega, z}$ as

$$
\cosh \left(z \alpha_{N}\right)+\frac{z^{2}}{2} \sum_{s=1}^{N-1} \omega_{s N} \alpha_{s}^{2} e^{z \alpha_{N}}=1+\frac{z^{2}}{2} h_{\omega, z}(\alpha) .
$$

Writing the flow action as

$$
\Phi_{i N}^{t}(\alpha)=\alpha^{\prime}
$$

we get

$$
\begin{aligned}
\alpha_{i}^{\prime} & =\frac{-\left[\sinh \left(z \alpha_{N}\right)-\frac{z^{2}}{2} \sum_{s=1}^{N-1} \omega_{s N} \alpha_{s}^{2} e^{z \alpha_{N}}\right] S_{\omega_{i N}}(t)+\left[z \alpha_{i} e^{z \alpha_{N}}\right] C_{\omega_{i N}}(t)}{z F_{i N}^{\omega, z}(\alpha, t)}, \\
\alpha_{N}^{\prime} & =\frac{1}{z} \ln F_{i N}^{\omega, z}(\alpha, t), \\
\alpha_{j}^{\prime} & =\frac{\alpha_{j} e^{z \alpha_{N}}}{F_{i N}^{\omega, z}(\alpha, t)}, \quad j \neq i, N .
\end{aligned}
$$

The limit $z \rightarrow 0$ can be obtained after considering the first order in $z$ of the function $F_{i N}^{\omega, z}$ :

$$
F_{i N}^{\omega, z}(\alpha, t)=1+z\left[\omega_{i N} S_{\omega_{i N}}(t) \alpha_{i}+C_{\omega_{i N}}(t) \alpha_{N}\right]+o\left(z^{2}\right),
$$

and this result yields the known linear flow, consisting of 'rotations' around the origin of the $i N$ plane,

$$
\alpha_{i}^{\prime}=C_{\omega_{i N}}(t) \alpha_{i}-S_{\omega_{i N}}(t) \alpha_{N}, \quad \alpha_{N}^{\prime}=\omega_{i N} S_{\omega_{i N}}(t) \alpha_{i}+C_{\omega_{i N}}(t) \alpha_{N}, \quad \alpha_{j}^{\prime}=\alpha_{j} .
$$

\section{Example: $U_{z}\left(\mathfrak{i s o}_{\omega_{2}, \omega_{3}}(3)\right)$}

In the previous section $U_{z}\left(\mathfrak{i s o}_{\omega_{2}, \omega_{3}, \ldots, \omega_{N}}(N)\right)$ has been studied, now we consider the particular case $N=3$. The following discussion clarifies the concepts introduced till now due to the 3 -dimensional nature of the group $T_{z 3}$. It is possible to represent graphically all the geometric constructions (an enlarged version of this paper with some figures can be sent under request to the authors).

The Hopf algebra $U_{z}\left(\mathfrak{i s o}_{\omega_{2}, \omega_{3}}(3)\right)$ is generated by $P_{1}, P_{2}, P_{3}, J_{12}, J_{13}$ and $J_{23}$. The commutators and the rest of structure tensors are obtained after setting the corresponding expressions of the previous section for $N=3$. In this case $U_{z}\left(\mathfrak{i s o}_{\omega_{2}, \omega_{3}}(3)\right)=U_{z}\left(\mathfrak{s o}_{\omega_{2}, \omega_{3}}(3)\right) \triangleright \triangleleft F\left(T_{z, 3}\right)$, where the group $T_{z, 3}$ is characterized by the composition law

$$
\left(\alpha_{1}^{\prime}, \alpha_{2}^{\prime}, \alpha_{3}^{\prime}\right)\left(\alpha_{1}, \alpha_{2}, \alpha_{3}\right)=\left(\alpha_{1}^{\prime}+e^{-z \alpha_{3}^{\prime}} \alpha_{1}, \alpha_{2}^{\prime}+e^{-z \alpha_{3}^{\prime}} \alpha_{2}, \alpha_{3}^{\prime}+\alpha_{3}\right)
$$

The translation generators constitute a system of global coordinates over $T_{z, 3}$

$$
P_{1}\left(\alpha_{1}, \alpha_{2}, \alpha_{3}\right)=\alpha_{1}, \quad P_{2}\left(\alpha_{1}, \alpha_{2}, \alpha_{3}\right)=\alpha_{2}, \quad P_{3}\left(\alpha_{1}, \alpha_{2}, \alpha_{3}\right)=\alpha_{3} .
$$


Respect to these coordinates the action of $S O_{\omega_{2}, \omega_{3}}(3)$ over $T_{z, 3}$, induced by the structure of $U_{z}\left(\mathfrak{s o}_{\omega_{2}, \omega_{3}}(3)\right)$-algebra module of $F\left(T_{z, 3}\right)$, is given by the vector fields

$$
\begin{aligned}
& \hat{J}_{12}=-P_{2} \frac{\partial}{\partial P_{1}}+\omega_{12} P_{1} \frac{\partial}{\partial P_{2}}, \\
& \hat{J}_{13}=-\left[\frac{1-e^{-2 z P_{3}}}{2 z}+\frac{z}{2}\left(\omega_{13} P_{1}^{2}-\omega_{23} P_{2}^{2}\right)\right] \frac{\partial}{\partial P_{1}}-z \omega_{13} P_{1} P_{2} \frac{\partial}{\partial P_{2}}+\omega_{13} P_{1} \frac{\partial}{\partial P_{3}}, \\
& \hat{J}_{23}=-z \omega_{23} P_{2} P_{1} \frac{\partial}{\partial P_{1}}-\left[\frac{1-e^{-2 z P_{3}}}{2 z}+\frac{z}{2}\left(-\omega_{13} P_{1}^{2}+\omega_{23} P_{2}^{2}\right)\right] \frac{\partial}{\partial P_{2}}+\omega_{23} P_{2} \frac{\partial}{\partial P_{3}} .
\end{aligned}
$$

The (generalized) distribution generated by these fields is integrable since they close the algebra $\mathfrak{s o}_{\omega_{2}, \omega_{3}}(3)$. The invariant

$$
h_{\omega, z}=\omega_{13} P_{1}^{2} e^{z P_{3}}+\omega_{23} P_{2}^{2} e^{z P_{3}}+\left[\frac{\sinh \left(\frac{z}{2} P_{3}\right)}{\frac{z}{2}}\right]^{2}
$$

allows us to analyze easily the nature of the leaves of the foliation. The 2-dimensional leaves correspond to the connected components of the sets $h_{\omega, z}^{-1}(t) \subset T_{z, 3}$, being $t \in \mathbb{R}$ a regular value of $h_{\omega, z}$. For example, when $\left(\omega_{2}>0, \omega_{3}>0 ; z>0\right)$ two strata appear: the origin point and the rest of the space. In the non-deformed case the study is reduced essentially to classify the family of quadrics

$$
\omega_{13} \alpha_{1}^{2}+\omega_{23} \alpha_{2}^{2}+\alpha_{3}^{2}+c=0 .
$$

When $c \neq 0$ every connected component constitutes a 2-dimensional orbit of the action, but for $c=0$ zero-dimensional orbits appear.

Last expressions (3.2) and (3.15) allow to describe the oneparameter flows associated to the generators. For $\hat{J}_{12}$ it is obtained a linear action

$$
\Phi_{12}^{t}\left(\alpha_{1}, \alpha_{2}, \alpha_{3}\right)=\left(C_{\omega_{12}}(t) \alpha_{1}-S_{\omega_{12}}(t) \alpha_{2}, \omega_{12} S_{\omega_{12}}(t) \alpha_{1}+C_{\omega_{12}}(t) \alpha_{2}, \alpha_{3}\right),
$$

unlike that happens for $\hat{J}_{13}$ and $\hat{J}_{23}$

$$
\begin{aligned}
\Phi_{13}^{t}\left(\alpha_{1}, \alpha_{2}, \alpha_{3}\right)= & \left(\frac{-\left[\sinh \left(z \alpha_{3}\right)+\frac{z^{2}}{2}\left(\omega_{13} \alpha_{1}^{2}+\omega_{23} \alpha_{2}^{2}\right) e^{\left.z \alpha_{3}\right]} S_{\omega_{13}}(t)+z \alpha_{2} C_{\omega_{13}}(t)\right.}{z F_{13}(\alpha, t)},\right. \\
\Phi_{23}^{t}\left(\alpha_{1}, \alpha_{2}, \alpha_{3}\right)= & \left(\frac{\alpha_{2} e^{z \alpha_{3}}}{F_{13}(\alpha, t)}, \frac{1}{z} \ln F_{13}(\alpha, t)\right) \\
& \left.\quad \frac{-\left[\sinh \left(z \alpha_{3}\right)+\frac{z^{2}}{2}\left(\omega_{13} \alpha_{1}^{2}+\omega_{23} \alpha_{2}^{2}\right) e^{z \alpha_{3}}\right] S_{\omega_{23}}(t)+z \alpha_{2} C_{\omega_{23}}(t)}{z F_{23}(\alpha, t)}, \frac{1}{z} \ln F_{23}(\alpha, t)\right),
\end{aligned}
$$


where

$$
\begin{aligned}
F_{i 3}(\alpha, t)=\left[\cosh \left(z \alpha_{3}\right)+\frac{z^{2}}{2}\left(\omega_{13} \alpha_{1}^{2}+\omega_{23} \alpha_{2}^{2}\right) e^{z \alpha_{3}}\right] \\
\quad+\left[\sinh \left(z \alpha_{3}\right)-\frac{z^{2}}{2}\left(\omega_{13} \alpha_{1}^{2}+\omega_{23} \alpha_{2}^{2}\right) e^{z \alpha_{3}}\right] C_{\omega_{i 3}}(t)+z \omega_{i 3} \alpha_{i} e^{z \alpha_{3}} S_{\omega_{i 3}}(t) .
\end{aligned}
$$

The action of the oneparameter subgroups gives a new foliation of the 2-dimensional leaves presented in the previous subsection.

The curves that appear in the foliation due, for example, to $\hat{J}_{13}$ may be interpreted as the intersection of the surfaces determined by the invariants

$$
\omega_{13} P_{1}^{2} e^{z P_{3}}+\omega_{23} P_{2}^{2} e^{z P_{3}}+\left[\frac{\sin \left(\frac{z}{2} P_{3}\right)}{\frac{z}{2}}\right]^{2}, \quad P_{2} e^{z P_{3}} .
$$

Summarizing, all the qualitative characteristics relative to the deformation with respect to the flow of the fields $\hat{J}_{i j}$, appear in the case $N=3$.

\section{Concluding remarks}

It is worthy to note that the reinterpretation of the bicrossproduct structure $\mathcal{H}=U(\mathcal{K}) \bowtie U_{z}(\mathcal{L})$, in the case that $U_{z}(\mathcal{L})$ is commutative (but noncocommutative) Hopf algebra, as $H=U(\mathcal{K}) \bowtie$ Fun $\left(L_{z}\right)$ allows to carry the action determining the bicrossproduct to an action of the group $K$ on $L_{z}$.

For the algebras involved in this work, in the deformed case, i.e, $z \neq 0$, the above mentioned action is local and nonlinear although in the opposite case the action is global and linear.

The flows have been obtained studying the case of $\omega_{i}>0$. An analytical dependence of the flow on the parameters $\omega_{i}$ is observed, which makes unnecessary to repeat the computations for the other values of the $\omega_{i}$ 's. This result is very interesting since the structure of the orbit space of the action of $\left.S O_{\omega_{2}, \omega_{3}, \ldots, \omega_{N}}(N)\right)$ on $T_{z, N}$ is very complicated, which difficulties to obtain directly the flows for each particular case.

In [5] the flows have been used for the computation of the induced representations for the $U_{z}\left(\mathfrak{i s o}_{\omega}(2)\right)$. For higher dimensions the problem of constructing the induced representations is very cumbersome and it is still an open problem.

The CK family $U_{z}\left(\mathfrak{i s o}_{\omega_{2}, \omega_{3}}(3)\right)$ contains, for instance, the $q$-Poincaré algebra $\left(\omega_{2}<0, \omega_{3}>0\right)$, $\left(\omega_{2}>0, \omega_{3}<0\right),\left(\omega_{2}<0, \omega_{3}<0\right)$, the $q$-Galilei algebra $\left(\omega_{2}=0, \omega_{3}>0\right)$ and the $q$-Euclidean algebra $\left(\omega_{2}>0, \omega_{3}>0\right)$. For a physical meaning of their generators see Ref. [11].

We finish with the following remarks about the systems (3.10):

1) The second order systems associated to (3.10),

$$
\ddot{x}=-a x \dot{x}-a\left(1+2 x+a x^{2}\right) x, \quad \ddot{y}=-\frac{1}{2} \dot{y}^{2}-\frac{1}{2} a\left(1+b e^{-2 y}\right),
$$

can be interpreted in both cases as moving objects over a straight line under the action of forces depending on the position and the velocity. 
2) The system (3.10) is associated to the vector field over $\mathbb{R}^{2}$

$$
X_{a, b}=\left[-\frac{1}{2} a x^{2}-\frac{1}{2}+\frac{1}{2} b e^{-2 y}\right] \frac{\partial}{\partial x}+a x \frac{\partial}{\partial y},
$$

which admits a hamiltonian description as we are going to prove. Obviously, the pair $(x, y)$ is not a chart of canonical coordinates since the 1-form obtained by contraction of the vector field and the symplectic 2-form associated to this chart $\left.\left(X_{a, b}\right\rfloor(d x \wedge d y)\right)$ is not exact. Hence, let us consider a general symplectic 2 -form $\omega=\Omega d x \wedge d y$, with $\Omega$ to be determined. Since $h_{a, b}$ is an invariant of the system it is evident that the Hamiltonian of the system has to be of the form $h=f \circ h_{a, b}$, with $f: \mathbb{R} \rightarrow \mathbb{R}$, which is not univocally determined. The vector field associated to $h$ by means of the symplectic structure is fixed by $\left.X_{h}\right\rfloor \omega=-d h$. So,

$$
X_{h}=-\frac{f^{\prime} \circ h_{a, b}}{\Omega} \partial_{y} h_{a, b} \frac{\partial}{\partial x}+\frac{f^{\prime} \circ h_{a, b}}{\Omega} \partial_{x} h_{a, b} \frac{\partial}{\partial y} .
$$

Identifying $X_{h}$ with $X_{a, b}$ two equations are obtained, but only one is independent. Hence,

$$
\Omega=f^{\prime} \circ h_{a, b} \frac{\partial_{x} h_{a, b}}{a x}=2 e^{y} f^{\prime} \circ h_{a, b} .
$$

The simple choice $f(t)=t$ allows us to obtain the 2 -form $\omega=2 e^{y} d x \wedge d y$, that is independent of the parameters $a$ and $b$. With the above election of $f$ the Hamiltonian of $X_{a, b}$ is the invariant $h_{a, b}$.

\section{Acknowledgments}

This work has been partially supported by DGES of the Ministerio de Educación y Cultura de España under Project PB98-0360, and the Junta de Castilla y León (Spain).

\section{References}

[1] O. Arratia and M.A. del Olmo in Anales de Física, Monografías, vol. 5 (Ciemat/RSEF, Madrid, 1998); math.QA/0110265.

[2] O. Arratia and M.A. del Olmo in Publicaciones de la Real Sociedad Matemática Española, vol. 1, E. Fuster and J. Monterde edit. (RSME, Madrid, 2000); math.QA/0110266.

[3] O. Arratia and M.A. del Olmo, J. Math. Phys. 41, 4817 (2000).

[4] O. Arratia and M.A. del Olmo, Representations of quantum bicrossproduct algebras. Preprint, UVA 2001; math.QA/0110275.

[5] O. Arratia and M.A. del Olmo, Induced Representations of Quantum Kinematical Algebras and Quantum Mechanics; math.QA/0202101 (to be published in J. Phys. A).

[6] S. Majid, J. Alg. 130 (1990) 17; Isr. J. Math. 72 (1990), 133; Foundations of quantum group theory (Cambridge Univ. Press, Cambridge 1995). 
[7] M. Santander, F.J. Herranz and M.A. del Olmo, Kinematics and homogeneous spaces for symmetrical contractions of orthogonal groups in "Group Theoretical Methods in Physics", pp. 455. Eds. M. A. del Olmo, M. Santander and J. Mateos-Guilarte, Anales de Física, Monografías, 1 Vol. I (CIEMAT/RSEF, Madrid 1993).

[8] F.J. Herranz, M. de Montigny, M.A. del Olmo and M. Santander, J. Phys. A: Math. Gen. 27 (1994), 2515.

[9] J.A. de Azcárraga, M. A. del Olmo, J. C. Pérez Bueno and M. Santander, J. Phys. A: Math. Gen. 30 (1997), 3069.

[10] A. Ballesteros, F.J. Herranz, M.A. del Olmo and M. Santander, J. Phys. A: Math. Gen. 26 (1993), 5801.

[11] A. Ballesteros, F.J. Herranz, M.A. del Olmo and M.Santander, J. Phys. A: Math. Gen. 27 (1994), 1283.

[12] V. Chari and A. Pressley, A guide to quantum groups (Cambridge Univ. Press, Cambridge 1994).

[13] A. Ballesteros, F.J. Herranz, M.A. del Olmo and M. Santander, J. Math. Phys. 35 (1994), 4928.

[14] A. Ballesteros, F.J. Herranz, M.A. del Olmo and M. Santander, Lett. Math. Phys. 33 (1995), 273. 